Research Paper

\title{
Hypoxia-inducible factor-1 alpha Correlates with Tumor-Associated Macrophages Infiltration, Influences Survival of Gastric Cancer Patients
}

\author{
Wei-jie Zhang1,2\#, Cheng Chen ${ }^{6 \#, ~ Z h i-h u a ~ Z h o u ~}{ }^{7 \#, ~ S h a n-t i n g ~ G a o ², ~ T e o n g ~ J i n ~ T e e 3, ~ L i u-q i n g ~ Y a n g 4, ~}$

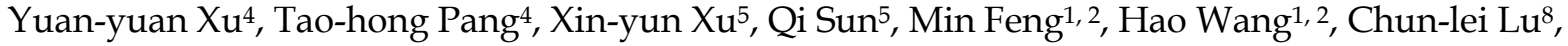 \\ Guo-zhong $\mathrm{Wu}^{8}$, Sheng $\mathrm{Wu}^{8}$, Wen-xian Guan ${ }^{1,2}$, Gui-fang $\mathrm{Xu}^{4 \bowtie}$ \\ 1. Department of General surgery, Drum Tower Clinical College of Nanjing Medical University, Nanjing, Jiangsu Province, China; \\ 2. Department of General surgery, Affiliated Drum Tower Hospital of Nanjing University Medical School, Nanjing, Jiangsu Province, China; \\ 3. Department of Medical, Gastroenterology unit, Nilai Medical Center, Nilai, Negeri Sembilan, Malaysia. \\ 4. Department of Gastroenterology, Affiliated Drum Tower Hospital of Nanjing University Medical School, Nanjing, Jiangsu Province, China; \\ 5. Department of Pathology, Affiliated Drum Tower Hospital of Nanjing University Medical School, Nanjing, Jiangsu Province, China; \\ 6. Department of Radiotherapy, Jiangsu Cancer Hospital Affiliated to Nanjing Medical University, Nanjing, Jiangsu Province, China; \\ 7. Department of Pathology, 101th Hospital of PLA, Wuxi, Jiangsu Providence, China; \\ 8. Department of General surgery, 101th Hospital of PLA, Wuxi, Jiangsu Providence, China; \\ \# Wei-jie Zhang, Cheng Chen and Zhi-hua Zhou equally contributed to this study. \\ $\square$ Corresponding author: Gui-fang Xu, Email: 13852293376@163.com or Wen-xian Guan, Email: guan-wx@163.com \\ (1) Ivyspring International Publisher. This is an open access article distributed under the terms of the Creative Commons Attribution (CC BY-NC) license \\ (https://creativecommons.org/licenses/by-nc/4.0/). See http://ivyspring.com/terms for full terms and conditions.
}

Received: 2017.01.04; Accepted: 2017.05.10; Published: 2017.07.02

\begin{abstract}
Background: Hypoxia was a common feature for accelerating tumor metastasis by both inducting epithelial-mesenchymal transition (EMT) of tumor cells and polarization of tumor-associated macrophages (TAMs). The association and roles between hypoxia, EMT and TAMs in the biological behavior of gastric cancer $(\mathrm{GC})$ for the time being recurrence is unclear.

Material and methods: hypoixa by expression of hypoxia-inducible factor-1 alpha (HIF-1 $\alpha$ ), polarized functional status of infiltrated TAMs by immunohistochemical staining of CD68 and CD 163, and the expression of E-cadherin as EMT property had been evaluated in 236 patients consecutive with histologically confirmed GC. Clinical significance was assessed for all these patients.

Results: High expression of HIF-1 $\alpha$ was found in patients with aggressive features, especially for recurrent patients. High infiltration of TAMs and abnormal expression of EMT-marker were also related to aggressive characteristics and predicted poor prognosis in GC. Meanwwhile, there existed a significant correlation among expression of HIF-1 $\alpha$, infiltration of TAMs and EMT marker in GC tissues. Multivariate Cox analysis revealed that high expression of HIF-1 $\alpha$ combined TAMs infiltration were independent prognostic factors for disease-specific survival rate.

Conclusion: HIF-l $\alpha$ is an unfavorable indicator for prognosis, may promote tumor progression through the induction of EMT and establishment of a pro-tumor immunosuppressive microenvironment. Further investigation into the therapeutic effects of blocking hypoxia is possible a potential strategy for GC treatment.
\end{abstract}

Key words: Gastric cancer, hypoxia-inducible factor-1 alpha, tumor-associated macrophages, epithelial-mesenchymal transition, prognosis

\section{Introduction}

Cancer incidence and mortality is increasing in China. It was documented 4292,000 new cancer cases and 2814,000 cancer death in 2015 , and gastric cancer
(GC) is the second leading cause of cancer-related death [1]. In spite of the progress in the surgical treatment and chemotherapy, the outcome of GC 
patients was still poor due to tumor relapse and metastasis [2-4]. Hypoxia is well recognized as a common feature of malignant tumors and plays an important role in therapeutic resistance, recurrence and metastasis [5-8]. It regulates the biological behaviors of both tumor cells and infiltrated immune cells [9-13], including induction of epithelial-to-mesenchymal transition (EMT) of cancer cell, activation and polarization of tumor-associated macrophages (TAMs). Despite of recent studies, the association and roles between hypoxia, EMT and TAMs and the biological behavior of GC remain largely unknown.

Hypoxia-inducible factor- 1 alpha (HIF-1a), the key transcriptional regulator activated by hypoxia, is correlated to both overall and disease-free survivals in GC after gastrectomy. The over-expression of HIF-1a was found to be a useful independent prognostic bio-marker [14,15]. Based on our previously study, high infiltration of TAMs was related to aggressive behavior, and an independent poor prognostic factor for GC [16]. In pancreatic cancer, expression of HIF-1a was significantly correlated with Chemical Chemokines 2 (CCL2) at the tissue level, and CCL2, a classical chemokine that can effectively recruit monocytes/macrophages to tumor sites, was related to patient prognosis [17].

As hypoxia plays an important role both in EMT and TAMs, it may have a close relationship with tumorigenesis of GC. In our study, we hypothesize a close relation between EMT and TAMs related to hypoxia. High infiltration of TAMs induced by HIF-1a in tumor microenvironment might facilitate the progression of EMT, which is associated with the recurrence and poor prognosis of GC. The clinicopathological features of GC, risk factors to predict recurrence and prognosis were also analyzed.

\section{Materials and Methods}

\section{Clinical and tumor specimens}

A total of 236 consecutive gastric cancer tumor specimens with histological confirmed were collected from the Department of General surgery, Drum Tower Hospital and the 101th Hospital of PLA between 2007 and 2009. 150 male and 86 female were included in this study with age ranging from 20 to 84 years. Patients with preoperative anti-cancer therapy, distant metastasis, and who lost follow-up were all excluded from this study. All samples were pathologically evaluated by two independent gastrointestinal pathologists based on WHO criteria [18]. None of these cases received any preoperative chemotherapy or radiation therapy.

\section{Ethics statement}

This study was approved by the clinical research ethics committees of both hospitals, and complied with the Declaration of Helsinki. Signed informed consent was taken from all studies participants or relatives.

\section{Immunohistochemistry}

All the tissues specimens (4- $\mu \mathrm{m}$-thick) were fixed in neutral buffered formalin and embedded in paraffin wax. Tissue sections were placed on charged glass slides, and then were subjected to deparaffinization in xylene and rehydration in a graded series of ethanol. Antigen retrieval was performed using citrate buffer at $\mathrm{pH}$ 6.0. Immunohistochemical staining was performed with the mouse monoclonal anti-HIF-1a antibody (Abcam, Cambridge, MA, USA), anti-CD68 antibody (Abcam, Cambridge, MA, USA), anti-CD163 antibody (Abcam, Cambridge, MA, USA) and anti-E-cadherin antibody (Abcam, Cambridge, MA, USA). The streptavidin-peroxidase technique was used for the purpose of staining. The sections were stained with 3-3'-diamino-benzidine (DAB) solution for 5 minutes followed by counterstaining with hematoxylin. An irrelevant rabbit antiserum served as a negative control. Positive controls consisted of each staining run and consisted of GCs known to express each of the antigens.

\section{Evaluation of Immunohistochemical Analysis}

The evaluation of the indicator expression was independently assessed, respectively, by two experienced pathologists who were blinded to the clinical data. TAMs density identified by CD68 and CD163 expression was estimated (per $\mathrm{mm}^{2}$ ) under a light microscope (Carl Zeiss Axio Scope. A1 microscope) at high-power fields (HPFs, 400×) in 5 fields per case. The mean number of positive cells in each case was determined. Low density was defined as the number of positive cells smaller than the average; otherwise considered as high density.

The staining results of HIF-1a and E-cadherin were scored semiquantitatively as follow. The percentage of immunoreactive cells was scored on a scale of 0 to 4 from no staining as 0,1 to $10 \%$ of cells stained as score 1,11 to $50 \%$ as 2,51 to $80 \%$ as 3 , and 81 to $100 \%$ as 4 . The staining intensities were graded from 0 as negative, to 1 as weak, 2 as moderate, and 3 as strong, respectively. The immunohistochemical score (IHS) was finally obtained by calculating the product of the staining intensity and the positive cell percentage. Patents with a score of $0-5$ were regarded 
as low expression, and those with equal or more than 6 were considered as high expression.

\section{Statistical analysis}

The correlation between HIF-1a, CD68, CD163 and E-cadherin and clinicopathological features was analyzed by the chi-square test. The Spear-man's rank correlation coefficient was used for analyzing the association of CD68, CD163 and E-cadherin expression levels with HIF-1a expression levels. Overall survival (OS) and disease-specific survival (DSS) were the designated endpoints. The definition of OS was the interval between surgery and last visit or death of any causes. As for DSS, cancer-related deaths were counted for the estimation of the disease-specific survival whereas other deaths uncorrelated to gastric cancer were censored. Survival curves were estimated using the Kaplan-Meier method, and differences in survival distributions were evaluated by the log-rank test. For multivariate analysis, the prognostic factors were analyzed using Cox's proportional hazard model. $P$-values $<0.05$ were considered statistically significant. All the data were analyzed by SPSS software, version 17.0 (SPSS Inc., Chicago, IL, USA).

\section{Results}

\section{Expression of HIF- $1 \alpha$ and TAMs markers in cancer tissues with clinicopathologic features in gastric cancer}

The clinicopathologic features of gastric cancer patients and their correlation with the expression of HIF-1a, CD68 and CD163 were described in Table 1. For CD68 and CD163 IHC staining, immunoreactivity was observed primarily in the cytoplasm and membrane of tumor cells, while majority gastric mucosal tissues showed negative or weak immunoreactivity with CD44. High and low expressions of the above-mentioned proteins, according to typical immunohistochemical staining patterns, in gastric cancer and para-cancer normal tissues were shown in Figure 1.

Of the 236 total cases, high expression of HIF-1a was observed in the cytoplasm of tumor cells in 138 cases $(58.5 \%)$. The positive rate of HIF-1a expression in GC tissues with recurrence was $70.1 \%(61 / 87)$, significantly higher $(P<0.05)$ than that without recurrence $51.7 \%$ (77/149). Patients with larger tumor size, advanced TNM stage, positive vascular invasion, deeper invasion and positive of LN metastasis also demonstrated with higher HIF-1a expression. There was no correlation between HIF-1a and other pathological parameters. High expressions of CD68 and CD163, detected in 50.8\% (120/236) and 54.4\% $(129 / 236)$ cases of GC, respectively, were closely related to the aggressive behaviors, such as advanced TNM stage, nodal metastasis, lymphovascular invasion and so on. The expression of TAMs markers for patients with recurrence was obviously higher $(P=0.001)$ than that without recurrence.

\section{Association among hypoxia, EMT property and infiltration of TAMs}

In GC tissues, high expressions of neoplastic cells in GC were found in more than half cases for HIF-1a and TAMs markers (Table 2). It was noted that expression loss of epithelial marker frequencies in the 236 GC tissues were $64.0 \%$ for E-cadherin. High expression of HIF-1a was associated with high expressions of CD68 $(r=0.209, P=0.001), \quad$ CD163 $(r=0.141, P=0.017)$, and low expression of E-cadherin $(r=-0.298, P=0.005)$ respectively. As for recurrent GC tissues, we found almost exactly the same result. High infiltration of TAMs was correlated with a loss expression of E-cadherin $(r=-0.3920$ and $r=-0.561$, $P<0.001$ for CD68 and CD163, respectively) in the same specimens (Table 3).

\section{Combined HIF-1 $\alpha$, EMT with TAMs infiltration was associated with disease-specific survival in GC patients}

The ending time for follow-up was January 31, 2016. All the cases were followed up every three months during the first two years post-discharge and every six months thereafter. The follow-up period after surgery was ranging from 20 to 84 months. When the follow-up was over, 98 patients were alive including 86 cases without recurrence and additional 12 with tumor-relapse, while the remaining 138 were dead (63 died of other diseases without recurrence and 75 deceased due to tumor recurrence).

Survival curves were analyzed by the Kaplan-Meier method and compared using the log-rank test. The disease-specific survival (DSS) and overall survival (OS) rates for patients with GC were $56.6 \%$ and $41.5 \%$, respectively. Patients with high expression of HIF-1a have an unfavorable overall and disease-specific survivals compared to those with low expression $(P<0.001) \quad($ Table 4; Figure 2A-2B). Multivariate Cox analysis revealed that high infiltration of TAMs and loss expression of epithelial protein E-cadherin were also statistically correlated with poor DSS $(P<0.05)$ (Figure $2 \mathrm{C}-\mathrm{D})$. All in all, as seen in Table 4, high expression of HIF-1a combined TAMs infiltration were independent prognostic factors for DSS and OS rates $(P<0.05)$. 

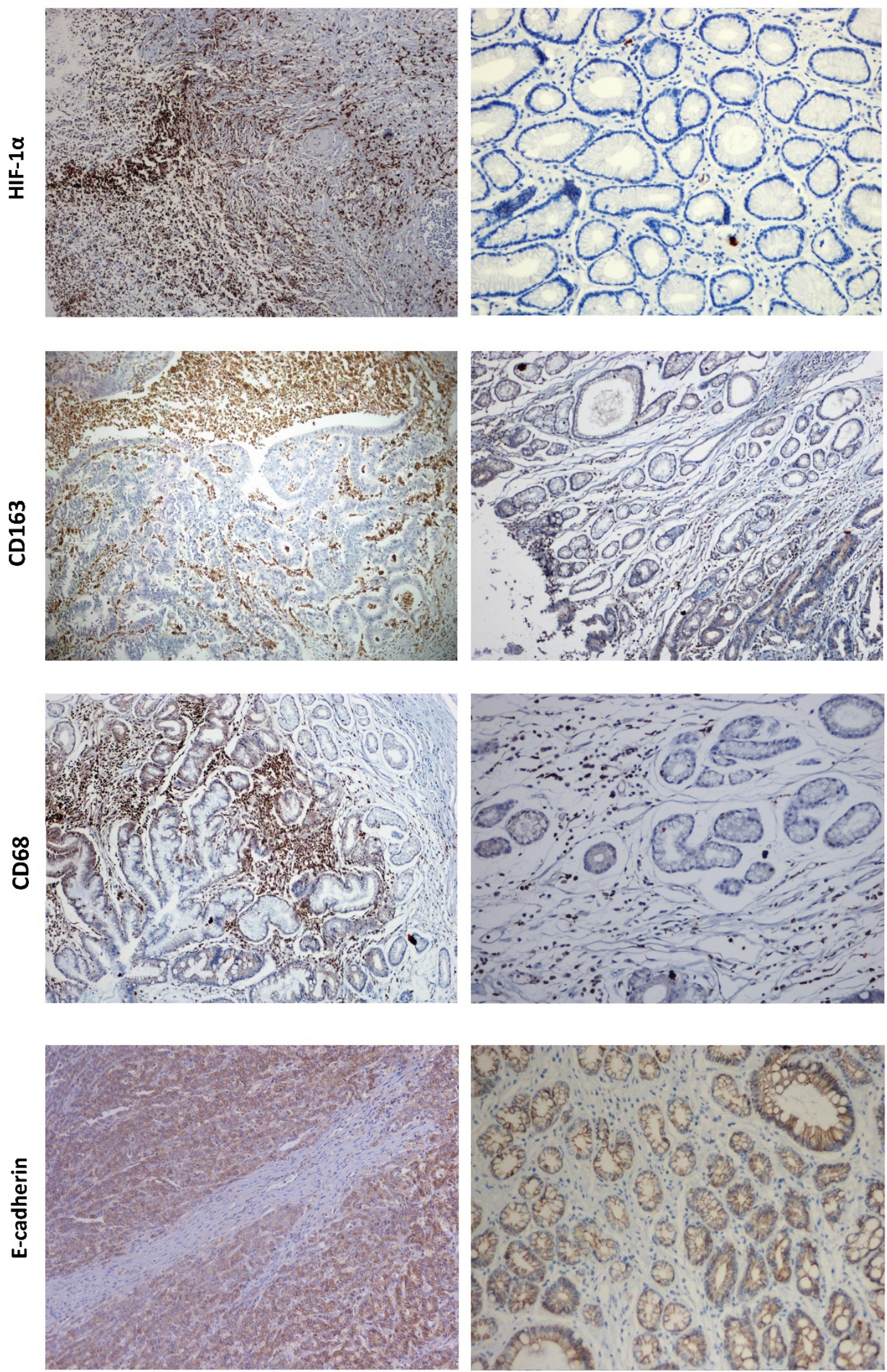

Gastric cancer tissue

Adjacent normal gastric tissue

Figure 1. Detection of levels of HIF-1 $\alpha$, TAMs markers and E-cadherin in GC tissue and adjacent normal tissue by IHC. Strong HIF-1 $\alpha$ immunoreactivity was identified in poorly differentiated cancer. Expression of TAMs markers was barely seen in normal tissue but was observed in GC tissue. E-cadherin was strongly positive membrane expression in adjacent normal gastric mucosa, but it was loss expression for most GC tissues. GC=gastric cancer, IHC=immunohistochemistry. 

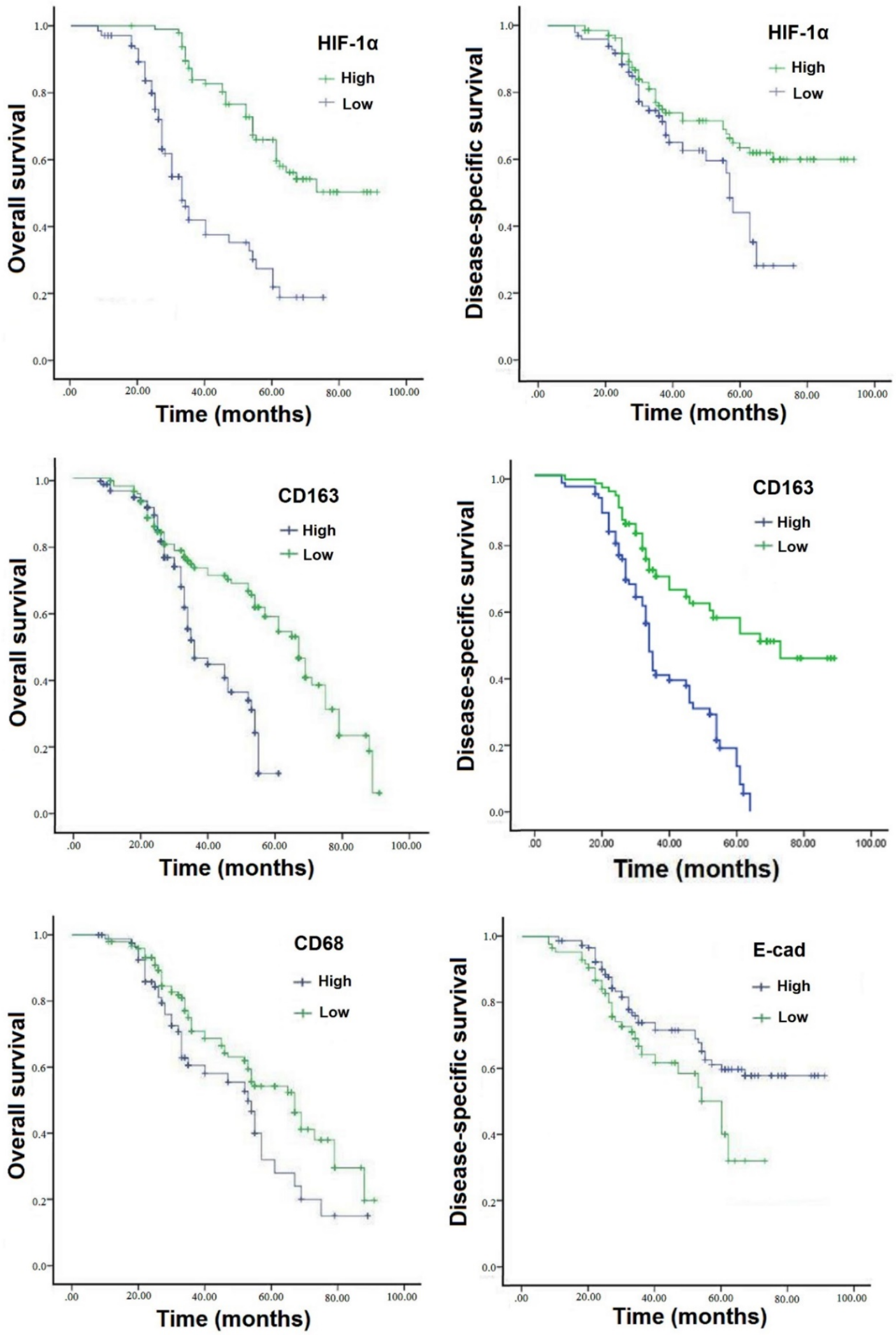

Figure 2. Expressions of HIF-1 $\alpha$, TAMs markers and associated EMT protein predict poor prognosis of GC. Patients with high expression of HIF-1 $\alpha$ demonstrated shorter DSS and OS than those with low expressed $(P<0.001)$. Patients with high expression levels of TAMs markers CD68 and CDI63 had a worse OS than those with low TAMs markers $(P<0.005)$, and patients with elevated levels of CDI63 also had a worse DSS. As for EMT marker, patients with loss expression of E-cadherin had a worse OS than those with high expression $(P<0.005)$. GC=gastric cancer, $\mathrm{DSS}=$ disease-specific survival, OS=overall survival. 
Table 1. The relationship between expression of HIF-1 $\alpha$, TAMs infiltration and clinicopathological features.

\begin{tabular}{|c|c|c|c|c|c|c|c|c|c|c|}
\hline Parameters & $\mathrm{N}$ & $\begin{array}{l}\text { HIF-1a } \\
\text { High (\%) }\end{array}$ & $\chi^{2}$ & $P$ value & $\begin{array}{l}\text { CD68 } \\
\text { High (\%) }\end{array}$ & $x^{2}$ & $P$ value & $\begin{array}{l}\text { CD163 } \\
\text { High (\%) }\end{array}$ & $x^{2}$ & $P$ value \\
\hline Age (yr) & & & 2.078 & 0.149 & & 1.443 & 0.230 & & 2.097 & 0.148 \\
\hline$<60$ & 129 & $70(54.3)$ & & & $61(47.3)$ & & & $65(50.4)$ & & \\
\hline$\geq 60$ & 107 & $68(63.6)$ & & & $59(55.1)$ & & & $64(59.8)$ & & \\
\hline Gender & & & 0.220 & 0.639 & & 1.681 & 0.195 & & 0.929 & 0.335 \\
\hline Male & 151 & $90(59.6)$ & & & $72(47.7)$ & & & $79(52.3)$ & & \\
\hline Female & 85 & $48(56.5)$ & & & $48(56.5)$ & & & $50(58.8)$ & & \\
\hline Differentiation & & & 2.979 & 0.087 & & 5.577 & 0.018 & & 4.734 & 0.030 \\
\hline Well/Moderate & 86 & $44(51.2)$ & & & $35(40.1)$ & & & $39(45.3)$ & & \\
\hline Poor & 150 & $94(62.7)$ & & & $85(56.7)$ & & & $90(60.0)$ & & \\
\hline Lauren type & & & 2.803 & 0.094 & & 0.586 & 0.444 & & 0.311 & 0.577 \\
\hline Intestinal & 110 & $58(52.7)$ & & & $53(48.2)$ & & & $58(52.7)$ & & \\
\hline Diffuse & 126 & $80(63.5)$ & & & $67(53.2)$ & & & $71(56.3)$ & & \\
\hline Tumor size $(\mathrm{cm})$ & & & 4.461 & 0.035 & & 3.750 & 0.053 & & 4.623 & 0.032 \\
\hline$<5$ & 99 & $50(50.5)$ & & & $43(43.4)$ & & & $46(46.5)$ & & \\
\hline$\geq 5$ & 137 & $88(64.2)$ & & & $77(56.2)$ & & & $83(60.6)$ & & \\
\hline T stage & & & 9.606 & 0.022 & & 12.508 & 0.006 & & 14.577 & 0.002 \\
\hline pT1 & 15 & $5(33.3)$ & & & $3(20.0)$ & & & $3(20.0)$ & & \\
\hline pT2 & 35 & 15 (42.9) & & & $12(34.3)$ & & & $13(37.1)$ & & \\
\hline pT3 & 88 & $54(61.4)$ & & & $47(53.4)$ & & & $52(59.1)$ & & \\
\hline pT4 & 98 & $64(65.3)$ & & & $58(59.2)$ & & & $61(62.2)$ & & \\
\hline Lymph node status & & & 7.979 & 0.005 & & 7.950 & 0.005 & & 5.089 & 0.024 \\
\hline N0/N1 & 77 & $35(45.5)$ & & & $29(37.7)$ & & & $34(44.2)$ & & \\
\hline $\mathrm{N} 2 / \mathrm{N} 3$ & 159 & $103(64.8)$ & & & $91(57.2)$ & & & $95(59.7)$ & & \\
\hline Vascular invasion & & & 4.286 & 0.038 & & 4.582 & 0.032 & & 4.408 & 0.036 \\
\hline Absent & 74 & $36(48.6)$ & & & $30(40.5)$ & & & $33(44.6)$ & & \\
\hline Present & 162 & $102(62.9)$ & & & $90(55.6)$ & & & $96(59.3)$ & & \\
\hline Recurrence & & & 12.661 & 0.006 & & 11.865 & 0.001 & & 11.377 & 0.001 \\
\hline Absent & 149 & 77 (51.7) & & & $63(42.3)$ & & & $69(46.3)$ & & \\
\hline Present & 87 & $61(70.1)$ & & & $57(65.5)$ & & & $60(69.0)$ & & \\
\hline TNM stage & & & 6.767 & 0.006 & & 8.627 & 0.003 & & 5.814 & 0.016 \\
\hline I-II & 80 & 37 (46.3) & & & $30(37.5)$ & & & $35(43.8)$ & & \\
\hline III-IV & 156 & $101(64.7)$ & & & $90(57.7)$ & & & $94(60.3)$ & & \\
\hline Serum CEA $(\mu \mathrm{g} / \mathrm{L})$ & & & 1.584 & 0.208 & & 1.630 & 0.202 & & 1.956 & 0.162 \\
\hline$<5$ & 90 & $48(53.3)$ & & & $41(45.6)$ & & & $44(48.9)$ & & \\
\hline$\geq 5$ & 146 & $90(61.6)$ & & & $79(54.1)$ & & & $85(58.2)$ & & \\
\hline
\end{tabular}

HIF-1a: hypoxia Inducible Factor-1 alpha; TNM: Tumor Node Metastasis; CEA: carcinoembryonic antigen.

Table 2. The association between HIF-1 $\alpha$ expression with TAMs infiltration and expression of E-cadherin in gastric cancer tissues

\begin{tabular}{|c|c|c|c|c|c|c|c|c|}
\hline \multirow[t]{2}{*}{ Parameters } & \multicolumn{2}{|c|}{$\begin{array}{l}\text { Overall } \\
\text { patients } \\
\text { HIF-1a }\end{array}$} & \multirow[t]{2}{*}{$r$} & \multirow[t]{2}{*}{$\begin{array}{l}P \\
\text { value }\end{array}$} & \multicolumn{2}{|c|}{$\begin{array}{l}\text { Recurrent } \\
\text { patients } \\
\text { HIF-1a }\end{array}$} & \multirow[t]{2}{*}{$r$} & \multirow[t]{2}{*}{$P$ value } \\
\hline & High & Low & & & High & Low & & \\
\hline CD68 & & & 0.209 & 0.001 & & & 0.266 & 0.013 \\
\hline High & 87 & 33 & & & 45 & 12 & & \\
\hline Low & 51 & 65 & & & 16 & 14 & & \\
\hline CD163 & & & 0.141 & 0.017 & & & 0.410 & $<0.001$ \\
\hline High & 90 & 39 & & & 49 & 10 & & \\
\hline Low & 48 & 59 & & & 12 & 16 & & \\
\hline E-cadherin & & & -0.298 & 0.005 & & & -0.463 & $<0.001$ \\
\hline High & 32 & 53 & & & 8 & 15 & & \\
\hline Low & 106 & 45 & & & 53 & 11 & & \\
\hline
\end{tabular}

\section{Discussion}

Tumor hypoxia is well recognized in oncology as a key factor resulting in treatment failure and poor prognosis [19-21]. Hypoxia is thought to play a pivotal role in accelerating tumor progression by both inducting EMT of tumor cells and polarization of TAMs [11]. Hypoxia promotes cancer progression by activating relevant gene expressions through hypoxia-inducible factors [20], and HIF-1a is found to be associated with a malignant behavior category, including postoperative prognosis [22].

The results from the present study supported the speculation that high expression of HIF-1a correlates with infiltration of TAMs, and contributes to DSS in GC. We found that hypoxia condition demonstrated as elevated level of HIF-1a was significant associated with infiltration of TAMs and EMT based on the expression of typical markers CD68, CD163 and E-cadherin, respectively. Moreover, both high expression of TAMs indicators and low expression of E-cadherin were significant correlated to high expression of HIF-1a in recurrent GC. High levels of HIF-1a was found to be associated with a malignant behavior category, including larger tumor size, advanced TNM stage, positive vascular invasion, deeper invasion and positive of LN metastasis. In multivariate Cox regression analysis, the results confirmed that the HIF-1a emerged as an independent prognostic factor for GC patients. 
Table 3. The association between infiltration of TAMs with expression of E-cadherin in gastric cancer tissues

\begin{tabular}{|c|c|c|c|c|c|c|c|c|}
\hline \multirow[t]{3}{*}{ Parameters } & \multicolumn{2}{|c|}{$\begin{array}{l}\text { Overall } \\
\text { patients }\end{array}$} & $r$ & \multirow[t]{3}{*}{$\begin{array}{l}P \\
\text { value }\end{array}$} & \multirow{2}{*}{\multicolumn{2}{|c|}{$\begin{array}{l}\begin{array}{l}\text { Recurrent } \\
\text { patients }\end{array} \\
\text { CD68 }\end{array}$}} & \multirow[t]{3}{*}{$r$} & \multirow[t]{3}{*}{$P$ value } \\
\hline & \multicolumn{2}{|c|}{ CD68 } & & & & & & \\
\hline & High & Low & & & High & Low & & \\
\hline E-cadherin & & & -0.392 & $<0.001$ & & & -0.388 & $<0.001$ \\
\hline High & 21 & 64 & & & 8 & 15 & & \\
\hline \multirow[t]{3}{*}{ Low } & 99 & 52 & & & 49 & 15 & & \\
\hline & \multicolumn{2}{|c|}{ CD163 } & & & \multicolumn{2}{|c|}{ CD163 } & & \\
\hline & High & Low & & & High & Low & & \\
\hline E-cadherin & & & -0.327 & $<0.001$ & & & -0.499 & $<0.001$ \\
\hline High & 28 & 57 & & & 7 & 16 & & \\
\hline Low & 101 & 50 & & & 53 & 11 & & \\
\hline
\end{tabular}

Table 4. Multivariate Cox regression analyses for overall and disease-specific survival of patients with gastric cancer

\begin{tabular}{lllllll}
\hline & \multicolumn{3}{l}{ Overall survival } & \multicolumn{4}{c}{ Disease-specific survival } \\
\cline { 2 - 7 } & HR & $95 \%$ CI & P value & HR & $95 \%$ CI & P value \\
\hline TNM stage & 2.497 & $1.091-5.713$ & $\mathbf{0 . 0 0 8}$ & 2.449 & $1.434-4.182$ & $\mathbf{0 . 0 0 2}$ \\
lymph node & 1.602 & $1.056-2.431$ & $\mathbf{0 . 0 3 5}$ & 1.875 & $1.191-2.952$ & $\mathbf{0 . 0 2 6}$ \\
status & & & & & & \\
HIF-1a & 2.395 & $1.033-5.551$ & $\mathbf{0 . 0 1 2}$ & 2.105 & $1.079-4.107$ & $\mathbf{0 . 0 1 0}$ \\
CD68 & 1.598 & $1.053-2.426$ & $\mathbf{0 . 0 4 1}$ & 1.393 & $1.023-1.897$ & 0.056 \\
CD163 & 1.774 & $1.206-2.610$ & $\mathbf{0 . 0 2 3}$ & 2.056 & $1.302-3.246$ & $\mathbf{0 . 0 1 6}$ \\
E-cadherin & 1.325 & $0.998-1.760$ & 0.065 & 1.524 & $1.099-2.113$ & $\mathbf{0 . 0 4 7}$ \\
\hline
\end{tabular}

In the current study, we also manifested that high expressions of TAMs markers, constant with over-expression of HIF-1a, were related to aggressive features of GC. the results also found a connection between TAMs and EMT in the tissues of GC, including patients with recurrence. Furthermore, multivariate Cox analysis also demonstrated that GC with high-expression of CD163 had a significant correlation with poor survival.

The mechanism by which EMT and TAMs interplay with each other under hypoxia in GC has not been clarified. The EMT plays a crucial role in GC development and progression [23] Accumulating evidence shows that the tumor microenvironment generates and sustains major EMT-triggering pathways for facilitating cancer cell morphological EMT-like changes and obtained the ability of invasion and migration under hypoxic conditions. Besides EMT induction, hypoxia is also thought to play an important role in the phenotypic control and promoting TAMs infiltration into tumor areas [12, 24-26]. In hepatocellular carcinoma, hypoxia can activate HIF-1a/CCL20/IDO axis signaling pathways, which is important for activating macrophages to establish an immunosuppressive tumor microenvironment [11]. Hypoxic TAMs, more likely to be M2-type macrophages, release factors to activate EMT-related signals, such as STAT3 or
TGF-beta1, to induction EMT and promoting tumor progression [27-30]. So, HIF-1a originated from cancer cells and tumor-related microenvironment may be one of the key promoters by which TAMs may modulate the progress of EMT in GC.

The results of the current study suggested that hypoxia and TAMs might be potential targets for development of immunotherapy of GC. Targeting HIF-1a or disrupting HIF-1a signal would block hypoxia-induced EMT and aggressive phenotype in cancer cells, with more efficacious and less toxic compounds to inhibit metastasis [31, 32]. Recent study, performed by Wang et al, found that macrophages promoted glioblastoma (GBM) invasion by the CCL4-CCR5 axis, and hypoxia enhanced the interaction between the two types of cells by up-regulating both CCL4 and CCR5 expression[33]; and for breast cancer, Song et al have demonstrated the HA-coated, mannan-conjugated $\mathrm{MnO} 2$ particle (Man-HA-MnO2) treatment significantly increased tumor oxygenation and down-regulated HIF-1a in the tumor, primed TAMs toward M1-like phenotype and enhanced chemotherapy response [34]. More intensive researches are needed to elucidate the mechanism by which cancer cells and macrophages interact with each other under hypoxia and verify the potential of hypoxia and TAMs as targets to battle cancer.

In summary, HIF-1a expression, correlated with malignant behavior, was increased in clinical GC specimens, especially in patients with recurrence, and it was associated with infiltration of TAMs, EMT induction and poor disease-specific survival. Therefore, the condition of anoxia and hypoxia could serve as a potential therapeutic strategy for exclusion of TAMs, deduction EMT and improve the prognosis ultimately.

\section{Acknowledgments}

This work was supported by grants from the National Natural Science Foundation of China (Grant Nos. 81201909, 81572338 and 81672380), the Fundamental Research Funds for the Central Universities (No. 021414380031), Nanjing Medical Science and Technology Development program (Nos. YKK12072, YKK15061 and YKK16078). This work was also a $C$ class sponsored project of Jiangsu provincial Six Talent Peaks (WSN-078).

\section{Competing Interests}

The authors declare no conflicts of interest.

\section{References}

1. Chen W, Zheng R, Baade PD, et al. Cancer statistics in China, 2015. CA Cancer J Clin. 2016; 66(2):115-32. 
2. Xu GF, Zhang WJ, Sun Q, et al. Combined epithelial-mesenchymal transition with cancer stem cell-like marker as predictors ofrecurrence after radical resection for gastric cancer. World J Surg Oncol. 2014;12:368.

3. Kim YJ, Kim SH, Kim JW, et al. Gastric cancer with initial bone metastasis: a distinct group of diseases with poor prognosis. Eur J Cancer. 2014; 50(16):2810-21.

4. Hirajima S, Komatsu S, Ichikawa D, et al. Liver metastasis is the only independent prognostic factor in AFP-producing gastric cancer. World J Gastroenterol. 2013; 19(36):6055-61.

5. Liang $\mathrm{Y}$, Zheng $\mathrm{T}$, Song $\mathrm{R}$, et al. Hypoxia-mediated sorafenib resistance can be overcome by EF24 through Von Hippel-Lindau tumor suppressor-dependent HIF-1a inhibition in hepatocellular carcinoma. Hepatology. 2013; 57(5):1847-57.

6. Hennessey D, Martin LM, Atzberger A, et al. Exposure to hypoxia following irradiation increases radioresistance in prostate cancer cells. Urol Oncol. 2013; 31(7):1106-16.

7. Wang $\mathrm{Y}$, Liu $\mathrm{X}$, Zhang $\mathrm{H}$, et al. Hypoxia-inducible IncRNA-AK058003 promotes gastric cancer metastasis by targeting $\gamma$-synuclein. Neoplasia. 2014; 16(12):1094-106.

8. Dekervel J, Hompes $\mathrm{D}$, van Malenstein $\mathrm{H}$, et al. Hypoxia-driven gene expression is an independent prognostic factor in stage II and III colon cancer patients. Clin Cancer Res. 2014; 20(8):2159-68.

9. Kohnoh T, Hashimoto N, Ando A, et al. Hypoxia-induced modulation of PTEN activity and EMT phenotypes in lung cancers. Cancer Cell Int. 2016; $16: 33$

10. Zhao X, Sun B, Liu Y, et al. Linearly Patterned Programmed Cell Necrosis Induced by Chronic Hypoxia Plays a Role in Melanoma Angiogenesis. J Cancer. 2016; 7(1):22-31.

11. Ye LY, Chen W, Bai XL, et al. Hypoxia-Induced Epithelial-to-Mesenchymal Transition in Hepatocellular Carcinoma Induces an Immunosuppressive Tumor Microenvironment to Promote Metastasis. Cancer Res. 2016; 76(4):818-30.

12. Guo $\mathrm{X}$, Xue $\mathrm{H}$, Shao $\mathrm{Q}$, et al. Hypoxia promotes glioma-associated macrophage infiltration via periostin and subsequent M2 polarization by upregulating TGF-beta and M-CSFR. Oncotarget. 2016 Sep 2. doi: 10.18632/oncotarget.11825.

13. Tripathi C, Tewari BN, Kanchan RK, et al. Macrophages are recruited to hypoxic tumor areas and acquire a pro-angiogenic M2-polarized phenotype via hypoxic cancer cell derived cytokines Oncostatin $M$ and Eotaxin. Oncotarget. 2014; 5(14):5350-68

14. Chen L, Shi Y, Yuan J, et al. HIF-1 alpha overexpression correlates with poor overall survival and disease-free survival ingastric cancer patients post-gastrectomy. PLoS One. 2014 Mar 10;9(3):e90678.

15. Isobe $\mathrm{T}$, Aoyagi $\mathrm{K}$, Koufuji $\mathrm{K}$, et al. Clinicopathological significance of hypoxia-inducible factor-1 alpha (HIF-1a) expression in gastriccancer. Int J Clin Oncol. $2013 ; 18(2): 293-304$

16. Zhang WJ, Zhou ZH, Guo M, et al. High Infiltration of Polarized CD163+ Tumor-Associated Macrophages Correlates with Aberrant Expressions of CSCs Markers, and Predicts Prognosis in Patients with Recurrent Gastric Cancer. J Cancer. 2017;8(3):363-370.

17. Li N, Li Y, Li Z, et al. Hypoxia Inducible Factor 1 (HIF-1) Recruits Macrophage to Activate Pancreatic Stellate Cells in Pancreatic Ductal Adenocarcinoma. Int J Mol Sci. 2016;17(6).

18. Bosman FT, Carneiro F, Hruban RH, et al. WHO Classification of Tumours of the Digestive System. IARC Press, 4th ed Lyon: 2010

19. Milosevic M, Warde P, Ménard C, et al. Tumor hypoxia predicts biochemical failure following radiotherapy for clinically localized prostate cancer. Clin Cancer Res. 2012; 18(7):2108-14.

20. Rankin EB, Giaccia AJ. Hypoxic control of metastasis. Science. 2016; 352(6282):175-80.

21. Applebaum MA, Jha $\mathrm{AR}, \mathrm{Kao} \mathrm{C}$, et al. Integrative genomics reveals hypoxia inducible genes that are associated with a poor prognosis in neuroblastoma patients. Oncotarget. 2016 Oct 17. doi: 10.18632/oncotarget.12713.

22. Zhang WJ, et al. Elevated expression of hypoxia inducible factor- 1 alpha is correlates to recurrence and poor outcome in gastric cancer. Int J Clin Exp Med. 2016; 9(4):7209-7216.

23. Peng Z, Wang CX, Fang EH, et al. Role of epithelial-mesenchymal transition in gastric cancer initiation and progression. World J Gastroenterol. 2014; 20(18):5403-10.

24. Tripathi C, Tewari BN, Kanchan RK, et al. Macrophages are recruited to hypoxic tumor areas and acquire a pro-angiogenic M2-polarized phenotype via hypoxic cancer cell derived cytokines Oncostatin $\mathrm{M}$ and Eotaxin. Oncotarget. 2014; 5(14):5350-68.

25. Huber R, Meier B, Otsuka A, et al. Tumour hypoxia promotes melanoma growth and metastasis via High Mobility Group Box-1 and M2-like macrophages. Sci Rep. 2016; 6:29914.

26. Henze AT, Mazzone M. The impact of hypoxia on tumor-associated macrophages. J Clin Invest. 2016; 126(10):3672-3679.

27. Wan S, Zhao E, Kryczek I, et al. Tumor-associated macrophages produce interleukin 6 and signal via STAT3 to promote expansion of human hepatocellular carcinoma stem cells. Gastroenterology. 2014; 147(6):1393-404.

28. Fan QM, Jing YY, Yu GF, et al. Tumor-associated macrophages promote cancer stem cell-like properties via transforming growth factor-beta1-induced epithelial-mesenchymal transition in hepatocellular carcinoma. Cancer Lett. 2014; 352(2):160-8
29. Jeong SK, Kim JS, Lee CG, et al. Tumor associated macrophages provide the survival resistance of tumor cells to hypoxic microenvironmental condition through IL-6 receptor-mediated signals. Immunobiology. 2017; 222(1):55-65.

30. Cui Y, Li YY, Li J, et al. STAT3 regulates hypoxia-induced epithelial mesenchymal transition in oesophageal squamous cell cancer. Oncol Rep. 2016; 36(1):108-16.

31. Liu T, Zhao L, Zhang Y, et al. Ginsenoside 20(S)-Rg3 targets HIF-1a to block hypoxia-induced epithelial-mesenchymal transition in ovarian cancer cells. PLoS One. 2014; 9(9):e103887.

32. Chou CC, Chuang HC, Salunke SB, et al. A novel HIF-1a-integrin-linked kinase regulatory loop that facilitates hypoxia-induced HIF-1a expression and epithelial-mesenchymal transition in cancer cells. Oncotarget. 2015;6(10):8271-85.

33. Song M, Liu T, Shi C, et al. Bioconjugated Manganese Dioxide Nanoparticles Enhance Chemotherapy Response by Priming Tumor-Associated Macrophages toward M1-like Phenotype and Attenuating Tumor Hypoxia. ACS Nano. 2016; 10(1):633-47.

34. Wang $\mathrm{Y}$, Liu $\mathrm{T}$, Yang $\mathrm{N}$, et al. Hypoxia and macrophages promote glioblastoma invasion by the CCL4-CCR5 axis. Oncol Rep. 2016; 36(6):3522-3528. 